\title{
The $\alpha$-Camera: A Quantitative Digital Autoradiography Technique Using a Charge-Coupled Device for Ex Vivo High-Resolution Bioimaging of $\alpha$-Particles
}

\author{
Tom Bäck and Lars Jacobsson
}

Department of Radiation Physics, the Sahlgrenska Academy at the University of Gothenburg, Gothenburg, Sweden

Bioconjugates used in internal radiotherapy exhibit heterogeneous distributions in organs and tumors, implying a risk of nonuniform dose distribution in therapeutic applications using $\alpha$-particle emitters. Tools are required that provide data on the activity distribution for estimation of absorbed dose on a suborgan level. The $\alpha$-camera is a quantitative imaging technique developed to detect $\alpha$-particles in tissues ex vivo. The aim of this study was to evaluate the characteristics of this imaging system and to exemplify its potential use in the development of $\alpha$-radioimmunotherapy. Methods: The $\alpha$-camera combines autoradiography with a scintillating technique and optical registration by a charge-coupled device (CCD). The imaging system characteristics were evaluated by measurements of linearity, uniformity, and spatial resolution. The technique was applied for quantitative imaging of ${ }^{211}$ At activity distribution in cryosections of tumors, kidney, and whole body. Intratumoral activity distributions of tumor-specific ${ }^{211} \mathrm{At}-\mathrm{MX} 35-\mathrm{F}\left(\mathrm{ab} \mathrm{b}_{2}\right)_{2}$ were studied at various times after injection. The postinjection activity distributions in the renal cortex and whole kidneys were compared for ${ }^{211} \mathrm{At}-\mathrm{F}\left(\mathrm{ab} \mathrm{b}^{\prime}\right)_{2}$ and ${ }^{211} \mathrm{At}-\mathrm{IgG}$ trastuzumab. Results: Quantitative analysis of $\alpha$-camera images demonstrated that the pixel intensity increased linearly with activity in the imaged specimen. The spatial resolution was $35 \pm 11 \mu \mathrm{m}$ (mean \pm SD) and the uniformity better than $2 \%$. Kidney cryosections revealed a higher cortex-to-whole kidney ratio for ${ }^{211} \mathrm{At}-\mathrm{F}\left(\mathrm{ab} \mathrm{b}^{\prime}\right)_{2}$ than for ${ }^{211} \mathrm{At}-\mathrm{lgG}(1.38 \pm 0.03$ and $0.77 \pm 0.04$, respectively) at $2 \mathrm{~h}$ after injection. Nonuniform intratumoral activity distributions were found for tumor-specific ${ }^{211} \mathrm{At}-\mathrm{MX} 35-\mathrm{F}\left(\mathrm{ab}^{\prime}\right)_{2}$ at $10 \mathrm{~min}$ and $7 \mathrm{~h}$ after injection; after $21 \mathrm{~h}$, the distribution was more uniform. Conclusion: The characteristics of the $\alpha$-camera are promising, suggesting that this bioimaging system can assist the development, evaluation, and refinement of future targeted radiotherapy approaches using $\alpha$-emitters. The $\alpha$-camera provides quantitative data on the activity distribution in tissues on a near-cellular scale and can therefore be used for small-scale dosimetry, improving the prediction of biologic outcomes with $\alpha$-particles with short path length and high linear energy transfer.

Key Words: ${ }^{211} \mathrm{At}$; $\alpha$-particles; autoradiography; radioimmunotherapy; imaging

J Nucl Med 2010; 51:1616-1623

DOI: 10.2967/jnumed.110.077578

Received Mar. 23, 2010; revision accepted Jul. 13, 2010

For correspondence or reprints contact: Tom Bäck, Department of Radiation Physics, the Sahlgrenska Academy at the University of Gothenburg, SE-413 45 Gothenburg, Sweden.

E-mail: tom.back@radfys.gu.se

COPYRIGHT $\odot 2010$ by the Society of Nuclear Medicine, Inc.
$\mathbf{P}$ romising results from preclinical studies $(1-6)$ and pilot clinical trials $(7,8)$ have increased the interest in clinical applications using $\alpha$-emitting radionuclides for the treatment of cancer. The high linear energy transfer (LET) of $\alpha$-particles yields a potent method of killing cancer cells. The short path length of $\alpha$-particles in tissues $(50-70 \mu \mathrm{m})$ is an advantage if the bioconjugate can be targeted specifically to tumor cells. In combination, the high LET and the short path length can make $\alpha$-particle therapy an effective treatment of micrometastases and small tumors.

Because of the short range of $\alpha$-particles and the heterogeneous distributions of the targeting molecules, the resulting dose distributions within organs and tumor tissues can be nonuniform. Estimations of the mean absorbed dose by whole organs and tumors may not accurately predict the biologic outcome of $\alpha$-particle therapies. Thus, micro- and small-scale dosimetry based on true radionuclide tissue distributions can be important tools to predict and explain normal-organ toxicities and tumor responses. Several studies (9-12) have emphasized the need for such tools.

We have developed a novel imaging technology for the ex vivo detection of $\alpha$-particles in tissues as a tool for assessing the suborgan activity distribution of an $\alpha$-emitting radionuclide. This technique provides rapid and quantitative imaging using a digital autoradiography setup and optical imaging of cryosections. Here we describe the establishment of this technique, provide examples of its application, and evaluate the characteristics of the imaging system. By injecting the $\alpha$-emitter ${ }^{211} \mathrm{At}$ in nude mice, either as free astatide or conjugated to antibodies, we obtained activity distribution images for several tissues including the kidneys, stomach, and tumor. As examples of quantitative analysis with the $\alpha$-camera, pilot experiments were conducted addressing 2 important issues of radioimmunoconjugates: tumor penetration versus time and renal distribution versus molecular size.

\section{MATERIALS AND METHODS}

\section{Description of Imaging System}

Principle of $\alpha$-Camera. The $\alpha$-camera is a digital autoradiography imaging technology using an optical registration of photons emitted from a scintillator dedicated to $\alpha$-particles. The geometric 
setup of the section sample during imaging is schematized in Figure 1. Cryosections of tissues (Fig. 1A) containing an $\alpha$-emitting radionuclide were transferred onto the scintillator (Fig 1B). Before being imaged optically with a charge-coupled device (CCD), the $\alpha$-particles (Fig. 1, black solid arrows) emitted in the tissue hit the scintillator and generate scintillation photons (Fig. 1, blue dotted arrows) that traverse the cryosection. The magnification scale of the images could be varied to approach the microscopic level using a macrophotographic setup. The acquired images allowed for quantitative analysis; pixel intensities were used to estimate the activity distribution of the radionuclide within the imaged cryosections.

Cryosectioning. Immediately after dissection, organs were placed in a cryomold containing cryoprotective gel. The molds were placed in an isopentane container and frozen using liquid nitrogen. The frozen tissues were placed in a cryostat microtome (HM 520; Microm International $\mathrm{GmbH}$ ) set to a temperature of $-19^{\circ} \mathrm{C}$. Sections $(10-16 \mu \mathrm{m})$ were cut and transferred to the top surface of a precut piece of the scintillating sheet for the imaging procedure. A consecutive cryosection, used for histologic comparison, was cut and transferred to a glass slide for hematoxylin and eosin (H\&E) staining.

Scintillator. Phosphor sheets (EJ-440; Eljen Technology), consisting of a silver-activated zinc sulfide phosphor (Fig. 1B) applied to 1 side of a clear polyester plastic sheet (Fig. 1C; $0.25 \mathrm{~mm}$ thick), were used for the scintillator. The phosphor layer (thickness, $\sim 0.06 \mathrm{~mm}$ ) density was sufficient to completely absorb common $\alpha$-particles. The photons emitted from the scintillator on $\alpha$-particle absorption had a peak wavelength of $450 \mathrm{~nm}$. Zinc sulfide was known as a scintillator already in 1903, when Crookes $(13,14)$ used his Spinthariscope to visualize individual scintillations caused by $\alpha$-particles.

Optical Setup and CCD. The imaging system (Supplemental Fig. 1; supplemental materials are available online only at http:// jnm.snmjournals.org) was assembled inside a dark box, minimizing ambient light artifacts. The optical setup consisted of a cooled high-sensitivity CCD coupled via an adapter to an optical lens

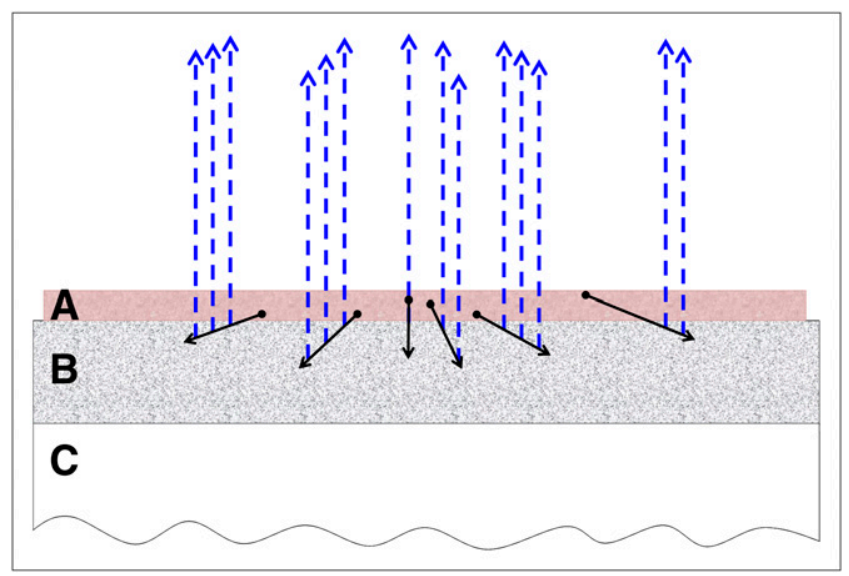

FIGURE 1. Schematized setup of sample configuration during imaging. Cryosections of tissues $(A)$ were placed on scintillating layer (B) that was coated on clear polyester sheet (C). $\alpha$-particles (black solid arrows) emitted in tissue hit scintillator and generate scintillation photons (blue dotted arrows), which traversed cryosection before being imaged. (60-mm f/2.8 Micro-Nikkor; Nikon Corp.) and an extension bellows (PB-6E; Nikon Corp.). The CCD was an SXV-H9 (Starlight Xpress Ltd.) equipped with the EXview HAD CCD progressive scan CCD image sensor (ICX285AL; Sony Corp.). The number of effective pixels was $1,392 \times 1,040$, and the pixel unit cell size was $6.45 \times 6.45 \mu \mathrm{m}$. The SXV-H9 detector used thermoelectric cooling to $30^{\circ} \mathrm{C}$ below ambient temperature, ensuring a low-noise readout. At a 450-nm wavelength, the spectral response was approximately $80 \%$. The specimens were imaged on an adjustable microscopy slide holder placed on an adjustable stand (Supplemental Figs. $1 \mathrm{E}$ and $1 \mathrm{~F}$, respectively).

Image Acquisition, Background Correction, and Analysis. Images were acquired (typically in a series of repeated exposures) in 16-bit data format using the MaxIm DL software (version 5.57; Diffraction Limited) and background-corrected using dark-frame subtraction. A series of dark frames was used for correction, using the same exposure time as used for the $\alpha$-images. The background-corrected images were summed, and the resulting combined images were filtered using a kernel median $(3 \times 3$ pixels $)$ filter to remove the noise from individual extreme pixels. For quantitative analysis, the images were imported into ImageJ (version 1.39; National Institutes of Health) or exported in 16-bit tagged image file format to the Advanced Image Data Analyzer software (version 4.22; Raytest $\mathrm{GmbH}$ ), where operator-defined or software-defined regions of interest (ROIs) were created for quantitative comparison of pixel intensity for different tissue compartments. The Advanced Image Data Analyzer software included a module (QWBA Image Overlay) for overlays with histologic images.

\section{Evaluation of Imaging System}

Linearity. To examine the radiation resistance, the scintillator was irradiated with $\alpha$-particles by applying an aqueous solution (50 MBq in $100 \mu \mathrm{L}$ ) of ${ }^{211}$ At directly onto the scintillating surface. The radioactive solution was evaporated to complete dryness. The retained initial activity on the scintillator surface was then imaged while decaying, by imaging the scintillation signal over time (repeated 60-s exposures). The same operator-defined ROI was identified for all the images in a series, and pixel intensities were plotted versus time. The linearity of the signal versus radioactivity was further evaluated at lower levels of activity using the same principle.

Uniformity. An aqueous solution of ${ }^{211} \mathrm{At}(20 \mathrm{MBq}$ in $5 \mathrm{~mL})$ in a Petri dish (10-cm diameter) served as a plane source to study the uniformity of the imaging system. A precut approximately $5 \times$ $5 \mathrm{~cm}$ piece of scintillator was placed $10 \mathrm{~mm}$ above the aqueous solution, with the scintillating surface facing the ${ }^{211}$ At solution. Uniformity was estimated from a central area of the imaged scintillator $(\sim 10 \times 10 \mathrm{~mm}, 500 \times 500$ pixels $)$, so that a uniform geometric distribution of the $\alpha$-particles within this area could be assumed. The scintillation signal was then imaged from the reverse side of the scintillator, allowing passage through the clear polyester plastic $(0.25 \mathrm{~mm})$ onto which the scintillating material was coated. The uniformity of the scintillation signal was evaluated by manually defining a series of ROIs of various sizes $(10 \times$ $10,25 \times 25,50 \times 50,75 \times 75$, and $100 \times 100$ pixels) side by side over the central area of the imaged scintillator piece. The relative SD was used as an estimate of the ROI-to-ROI uniformity by dividing the SD of the ROI mean by the mean total pixel intensity of the same ROI. The use of an aqueous solution as the plane source necessitated the reverse-side imaging and thus influenced 
the uniformity estimation due to the photon passage through the polyester plastic.

Pixel-to-Pixel Variation with Pixel Intensity. The influence of mean pixel intensity on the pixel-to-pixel intensity variations was studied, using the setup already described. Repeated exposures of the same decaying ${ }^{211}$ At sample were acquired at a decreasing rate of pixel intensity per second. The relative SD was calculated, using the same user-defined ROI $(300 \times 300$ pixels $)$ for all images and then related to the mean pixel intensity of the same ROI.

Spatial Resolution. The spatial resolution was estimated from exposures of radioactive line sources (thickness, $\sim 10 \mu \mathrm{m}$ ), derived from 10- $\mu \mathrm{m}$ kidney cryosections that were embedded in cryogel, sectioned again (perpendicular to the first sectioning), and placed on the scintillator. Hereby, the same section-to-scintillator geometry of the imaged line sources was the same as for the animal specimens.

Absorption of Scintillation Photons. The scintillation photons had to traverse the imaged cryosection before being registered. The absorption of the photons during tissue passage reduced the signal intensity. To investigate the absorption magnitude, a lighting box with a Kodak Wratten filter (no. 47, deep blue, 450 nm; Eastman Kodak Co.) was used as a light source. Exposures of light passing through a $16-\mu \mathrm{m}$ unstained kidney section (on a glass slide) were then related to matching exposures with an identical setup without the section. The absorption effect at $450 \mathrm{~nm}$ was estimated by dividing the images (pixel to pixel) from the 2 exposures.

Estimation of Activity from Quantitative Analysis. Radionuclide activity was estimated from imaged kidney sections (16 $\mu \mathrm{m})$ containing ${ }^{211}$ At. ROIs were software-defined, encompassing the whole kidneys. After imaging, the activity of each section was measured by $\gamma$-counting (Wizard 1480; Wallac Oy). The quantified ROI data (total pixel intensity per second) from each section was then related to the decay-corrected radionuclide activity.

\section{Applications of Imaging System}

Radionuclide and Labeling. The $\alpha$-particle-emitting radionuclide ${ }^{211}$ At was produced at the Cyclotron and PET Unit, Rigshospitalet, Denmark. The half-life of ${ }^{211} \mathrm{At}$ is $7.2 \mathrm{~h}$, and the emitted $\alpha$-particles $(7.45$ and $5.87 \mathrm{MeV}$ ) were generated through 2 branches of decay $(58.3 \%$ and $41.7 \%$, respectively). Dry distillation and radiolabeling was performed as described before $(15,16)$.

Monoclonal Antibodies and Cell Lines. The $\mathrm{F}\left(\mathrm{ab}^{\prime}\right)_{2}$ fragment of the monoclonal antibody MX35, directed against human epithelial ovarian cancer $(17,18)$, was produced by Strategic BioSolutions and developed and kindly provided by the Memorial Sloan-Kettering Cancer Center. Traztuzumab (Herceptin; Genentech) is a recombinant humanized monoclonal antibody directed against the extracellular domain of the HER2 protein. The human ovarian cancer cell line NIH:OVCAR-3 was obtained from the American Type Culture Collection.

Animals and Tissue Distribution Studies. The tissue distributions of ${ }^{211} \mathrm{At}$ and astatinated antibodies were studied in nude mice (age, 4-8 wk; BALB C nu/nu [Charles River]) after intravenous injections in the tail. At serial times after injection, the animals were sacrificed, and organs removed and prepared for imaging. All animals were maintained according to the ethical regulations of the Swedish Animal Welfare Agency.

Activity Distribution of ${ }^{211} A t-M X 35-F\left(a b^{\prime}\right) 2$ in Tumors. An ovarian carcinoma tumor model was used to investigate the intratumoral distribution of radiolabeled antibodies. Subcutaneous xenografts of the cell line NIH:OVCAR-3 were established as described before (2) and allowed 2 wk of growth. One to $10 \mathrm{MBq}$ $\left({ }^{211} \mathrm{At}-\mathrm{MX} 35-\mathrm{F}\left(\mathrm{ab}^{\prime}\right)_{2}\right)$ was injected, and at $10 \mathrm{~min}, 7 \mathrm{~h}$, and $21 \mathrm{~h}$ after injection the animals were sacrificed. These times were chosen to capture 3 different phases of intratumoral bioconjugate distribution: the vascular, capillary extravasation, and diffusionwith-binding phases (19). The tumors were dissected and cryosectioned as described. The sections $(10-16 \mu \mathrm{m})$ were imaged using repeated exposures that were later combined. Exposures were taken for $600,1,800$, and 3,600 s at $10 \mathrm{~min}$ after injection, $7 \mathrm{~h}$ after injection, and $21 \mathrm{~h}$ after injection, respectively. After imaging, the activity in each section was measured in a $\gamma$-counter. For each imaged section, a consecutive section was sectioned for $\mathrm{H} \& \mathrm{E}$. The acquired images were quantitatively analyzed by studying the pixel-intensity variations along a cross-sectional line profile over the tumor and from operator-defined ROIs over the whole tumor. This analysis was done by dividing each pixel value by the mean pixel intensity of the line profile or the entire ROI.

Renal Distribution of Bioconjugates with Different Molecular Sizes. Mice were injected (2-10 MBq) with astatinated IgG antibody (trastuzumab) or MX35-F $\left(\mathrm{ab}^{\prime}\right)_{2}$ fragments, and the renal activity distributions were compared at various times after injection (2-480 min after injection) by imaging (repeated 600-s exposures) of kidney cryosections $(10-16 \mu \mathrm{m})$. The images were combined and quantitatively analyzed by software-defined ROIs over the renal cortex and whole kidney. For each imaged kidney section, a cortex-to-whole kidney ratio was calculated by dividing the mean pixel intensity per area unit of the cortical ROI by the mean pixel intensity of the whole kidney ROI.

Distribution of Free Astatide in Whole-Body Sections. Elevated uptake of ${ }^{211}$ At has been noted in targeted $\alpha$-radioimmunotherapy in several organs, for example, thyroid, lung, spleen, and stomach (20-22). As a wider mapping of the distribution of free astatide, we used whole-body sectioning in combination with $\alpha$-camera imaging. Whole-body sectioning was performed (Active Biotech $\mathrm{AB})$ for 1 mouse using a technique dedicated to whole-body autoradiography $(23,24)$. Four hours after the intravenous injection of $10 \mathrm{MBq}$ of unbound ${ }^{211} \mathrm{At}$, the mouse was sacrificed, embedded in cryoprotective gel, and frozen in liquid nitrogen. Twenty-micron sections of the entire animal were cut at various levels in the sagittal plane and transferred onto the precoated $(30 \mu \mathrm{L}$, solution B) scintillator using the Macro-Tape-Transfer System Process (Instrumedics Inc.). The sections were placed in the cryostat and dried for $45 \mathrm{~min}$ before imaging.

\section{RESULTS}

\section{Evaluation of Imaging System}

Linearity. At high activity levels, plotting the pixel intensity data from the ROI of a repeated exposure series of a given sample revealed that, after an initial phase of saturated pixel intensity, the acquired signal was linear to activity over a time span corresponding to 2 half-lives of ${ }^{211}$ At (data not shown). No signal fading was detected. At lower activity levels, for pixel intensity versus time (Supplemental Fig. 2A) the pixel intensity was linear to activity, and from an exponential fit of the linear-logarithmic plot the decay of ${ }^{211}$ At was estimated to be $7.15 \mathrm{~h}$ (in good agreement with the actual half-life of $7.21 \mathrm{~h}$ ).

Uniformity. The uniformity was estimated to be below $2 \%$ for all ROIs studied using the relative SD as a measure. For ROIs of $10 \times 10,25 \times 25,50 \times 50,75 \times 75$, and $100 \times$ 

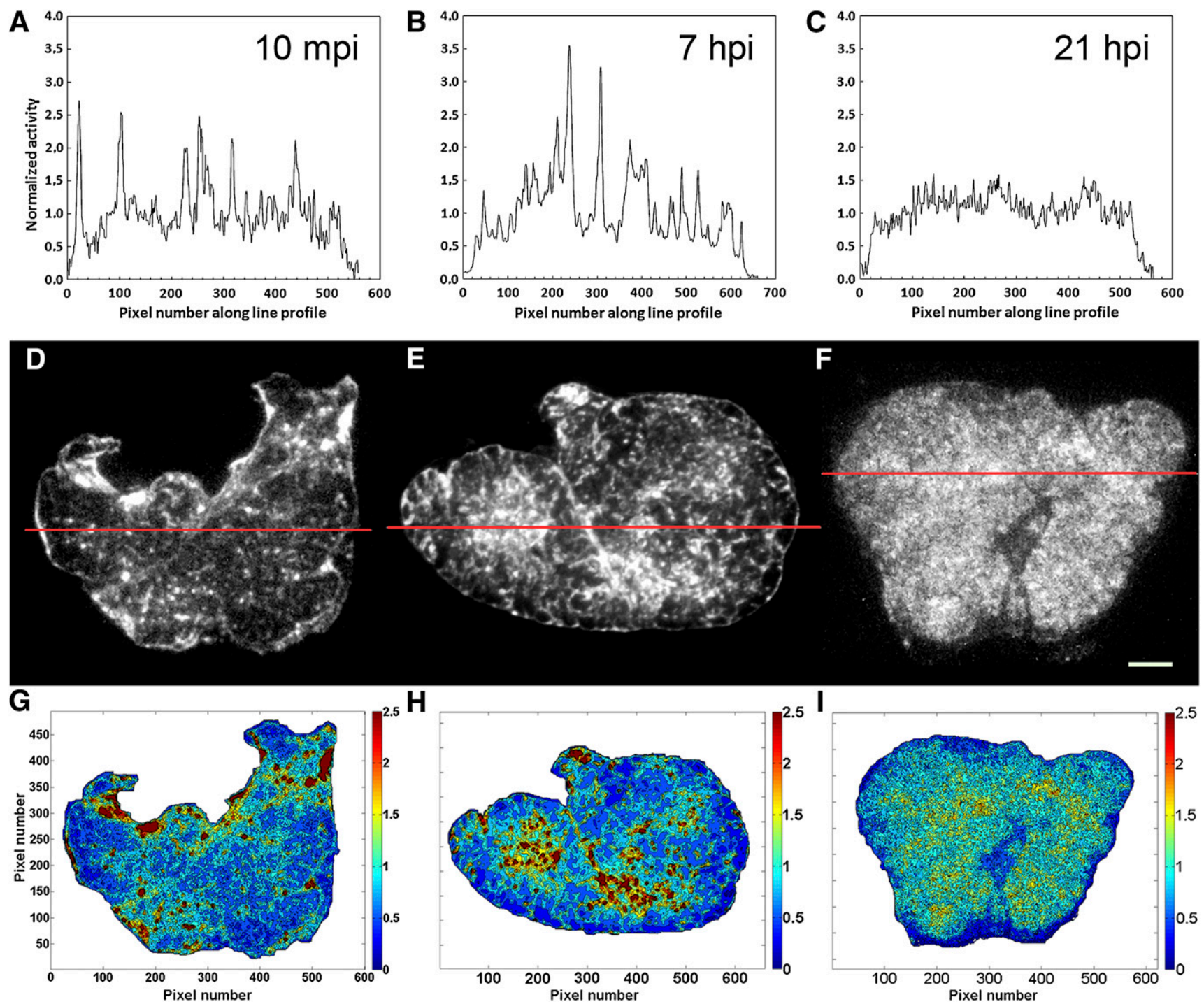

FIGURE 2. Activity distribution of tumor-specific ${ }^{211} \mathrm{At}-\mathrm{MX} 35-\mathrm{F}\left(\mathrm{ab}{ }^{\prime}\right)_{2}$ in OVCAR-3 xenografts. (A-C) Pixel intensity values (normalized to line profile mean pixel intensity) of line profile plotted as function of pixel number along line. (D-F) $\alpha-C a m e r a$ images taken $10 \mathrm{~min}, 7 \mathrm{~h}$, and $21 \mathrm{~h}$ after bioconjugate injection. Red lines mark line profile positions used in A-C. (G-I) Activity distribution histograms for whole tumor. Intensity value for each pixel was divided by mean pixel intensity of whole tumor. Normalized data were divided into 10 bins between 0 and 2.5, and each level was plotted in representative color described by color-code bar. White scale bar corresponds $1,000 \mu \mathrm{m}$ in D-F. hpi $=$ hours after injection; mpi $=$ minutes after injection.

100 pixels, the relative SDs were $1.84 \%, 1.29 \%, 1.14 \%$, $1.13 \%$, and $1.03 \%$, respectively.

Pixel-to-Pixel Variation with Pixel Intensity. The relative $\mathrm{SD}$ of the mean pixel-to-pixel variations was found to vary with total pixel intensity (Supplemental Fig. 2B), reaching a lower plateau value of approximately $2 \%$ when the maximum intensity level of the images was approached.

Spatial Resolution. The spatial resolution was estimated from 8 line profiles, and the calculated full width at half maximum was $35 \pm 11 \mu \mathrm{m}$ (mean $\pm \mathrm{SD}$ ). Supplemental Figure $3 \mathrm{~A}$ depicts 1 line profile with a corresponding full width at half maximum estimation of $26 \mu \mathrm{m}$.

Absorption of Scintillation Photons. The absorption of the scintillation photons during tissue passage reduced the signal intensity in direct relation to the thickness and optical density of the tissue section. To estimate this effect, we analyzed the variations in light transmission of an imaged $16-\mu \mathrm{m}$ whole-kidney section. The effect of absorption at $450 \mathrm{~nm}$ corresponded to a maximum-intensity variation of approximately $10 \%$. The effect of optical density variations can be corrected using a corresponding image of an adjacent section mounted on a glass slide.

Estimation of Radioactivity from Quantitative Analysis. We evaluated the ability of the $\alpha$-camera to estimate radioactivity in tissue sections by relating the quantified ROI data for an acquired image (16- $\mu \mathrm{m}$ kidney sections) to the measured radioactivity of the imaged sample. The ROI data (total pixel intensity per second in a whole-section 
ROI) from the imaged kidneys was plotted versus measured ${ }^{211}$ At activity in the same sample (Supplemental Fig. 3B). The pixel intensity values increased linearly with ${ }^{211} \mathrm{At}$ activity. The lowest detectable activity was not estimated, but activities of only $10-20 \mathrm{~Bq}$ of ${ }^{211} \mathrm{At}$ were sufficient to demonstrate the radionuclide distribution and permit quantitative analysis.

\section{Applications of Imaging System}

Activity Distribution of ${ }^{211} A t-M X 35-F\left(a b^{\prime}\right) 2$ in Tumors. Tumor images taken at various times after injection displayed marked differences in activity distribution (Fig. 2). The image at $10 \mathrm{~min}$ after injection revealed many small high-intensity areas that likely represent vasculature and capillaries (Fig. 2D). The larger high-intensity areas in the periphery of the tumor were most likely major blood vessels supporting the xenograft. In the image at $7 \mathrm{~h}$ after injection, the activity distribution resembled a glandular pattern, with small ring-shaped areas of higher intensity surrounding lower-intensity dark areas (Fig. 2E).

The tumor images were evaluated quantitatively by defining ROIs and line profiles. The activity distribution within the tumors was estimated from the normalized pixel intensities of the line profiles (Figs. 2A-2C), assuming a linear relation between image pixel intensity and activity in the imaged sections (Figs. 2D-2F). A 2-dimensional representation of the activity distribution (Figs. 2G-2I) showed activity histograms normalized to the ROI mean. As evident from the color-coded histograms (Figs. 2G-2I), the activity distribution was nonuniform at the first 2 time points (at $10 \mathrm{~min}$ and $7 \mathrm{~h}$ after injection), with small areas (red) 2.5-fold higher than the ROI mean interspersed among lower-activity areas (0.5-fold or less, dark blue). The heterogeneity may be even greater, as indicated by the unbinned line profile of the image at $7 \mathrm{~h}$ after injection (Fig. 2B); the highest peaks reached a factor of 3.5 above the mean. In contrast, the image at $21 \mathrm{~h}$ after injection (Figs. $2 \mathrm{C}, 2 \mathrm{~F}$, and 2I) depicted a more uniform activity distribution, with a large fraction of the tumor area displaying mean activity levels. When the sections at $7 \mathrm{~h}$ after injection were compared with the H\&E sections (Figs. 3A and 3B), the areas of high intensity were identified as stroma surrounding clusters of tumor cells, seen as darker areas, indicating that capillary extravasation of the bioconjugate had occurred $7 \mathrm{~h}$ after injection but that the diffusion with binding in central tumor areas was low. In contrast, the images taken at $21 \mathrm{~h}$ (Fig. 2F) showed a much more uniform activity distribution, indicating that the radionuclide was well distributed throughout all tumor compartments. The measured ${ }^{211} \mathrm{At}$ activities in the imaged sections ranged from 3 to $20 \mathrm{~Bq}$ (at start of exposure).

Renal Distribution of Bioconjugates with Different Molecular Sizes. To investigate the effect of molecular size of the bioconjugate on renal distribution in the kidney, we compared distributions of the astatinated $\mathrm{F}\left(\mathrm{ab}^{\prime}\right)_{2}$ antibody fragment and the IgG antibody. The activity distributions were similar after 20 min (Supplemental Figs. 4A and 4B), but there were clear differences at $2 \mathrm{~h}$ after injection (Supplemental Figs. 4C-4D). At $2 \mathrm{~h}$, the images disclosed a stronger retention of ${ }^{211} \mathrm{At}-\mathrm{MX} 35-\mathrm{F}\left(\mathrm{ab}^{\prime}\right)_{2}$ than of IgGantibody trastuzumab in the renal cortex. This difference was evaluated quantitatively by imaging at a range of times (10-480 min) after injection. A clear difference between $\mathrm{F}\left(\mathrm{ab}^{\prime}\right)_{2}$ and IgG emerged when the cortex-to-whole kidney ratio was studied versus time (Table 1 ). For all times studied, the ratios for the $\mathrm{F}\left(\mathrm{ab}^{\prime}\right)_{2}$ fragment were higher than for the IgG antibody. At $2 \mathrm{~h}$ after injection, the ratios (mean \pm SD) were $1.38 \pm 0.03$ and $0.77 \pm 0.04$ for ${ }^{211} \mathrm{At}-\mathrm{MX} 35-\mathrm{F}$ $\left(\mathrm{ab}^{\prime}\right)_{2}$ and ${ }^{211}$ At-trastuzumab, respectively.

Overall, the kidneys produced images with good morphologic visualization, presumably because of excellent perfusion and the high degree of tissue organization. The $\alpha$-camera captured images of the intrarenal distribution of ${ }^{211}$ At-trastuzumab at $30 \mathrm{~min}$ (Fig. 4A) and $2 \mathrm{~h}$ (Fig. 4B) after intravenous injection, visualizing small kidney structures such as glomeruli, medullary rays, and vascular branches stretching toward the renal cortex.

Distribution of Free Astatide in Whole-Body Sections. Free astatide distribution was investigated by imaging of whole-body sections. Four hours after intravenous injection of $10 \mathrm{MBq}$ of ${ }^{211} \mathrm{At}$, images of sections from different

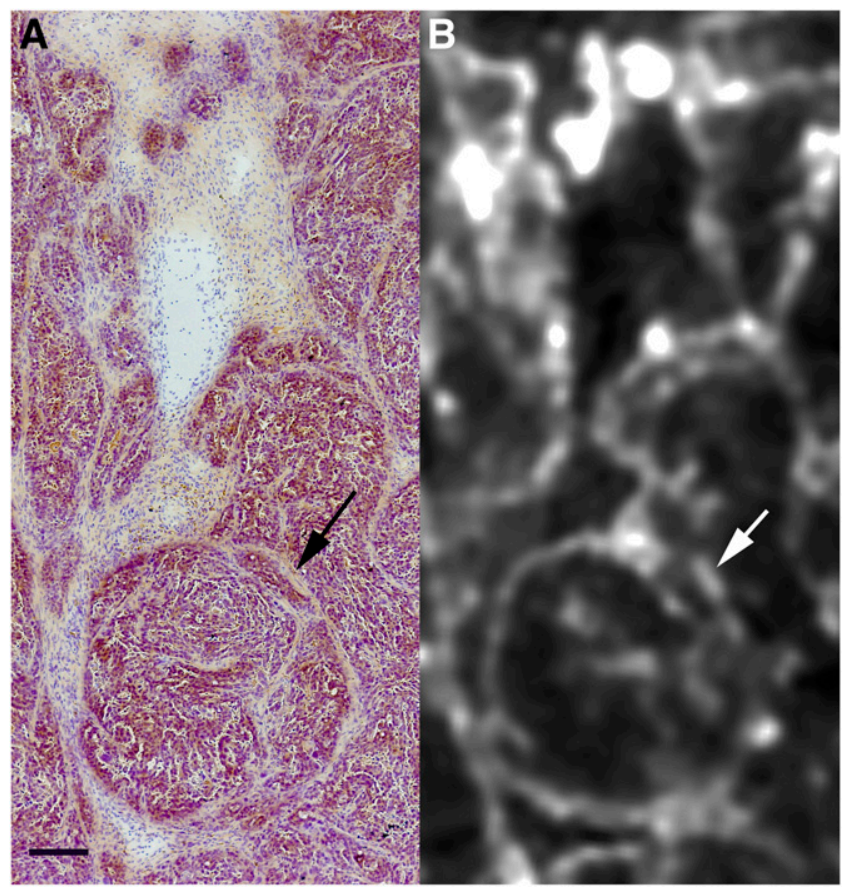

FIGURE 3. Overlay of $\alpha$-camera image with H\&E section. OVCAR-3 xenograft was imaged at $7 \mathrm{~h}$ after intravenous injection of tumor-specific ${ }^{211}$ At-MX35-F(ab') 2 . (A) Area of $\mathrm{H} \& \mathrm{E}$-stained section consecutive to $\alpha$-camera images is shown in Figure 4. (B) Same area seen in $\alpha$-camera image. White and black arrows indicate same region of stroma in tumor. Black bar indicates $200 \mu \mathrm{m}$. 


\begin{tabular}{|c|c|c|}
\hline \multirow{2}{*}{$\begin{array}{c}\text { Time after } \\
\text { injection (min) }\end{array}$} & \multicolumn{2}{|c|}{ Administered bioconjugate } \\
\hline & ${ }^{211} \mathrm{At}^{-\operatorname{lgG}{ }^{*}}$ & $\left.{ }^{211} \mathrm{At}-\mathrm{F}(\mathrm{ab})^{\prime}\right)_{2}^{\dagger}$ \\
\hline 10 & NA & $1.76 \pm 0.18$ \\
\hline 20 & $1.44 \pm 0.06$ & $1.56 \pm 0.01$ \\
\hline 30 & NA & $1.53 \pm 0.05$ \\
\hline 120 & $0.77 \pm 0.04$ & $1.38 \pm 0.03$ \\
\hline 270 & $0.91 \pm 0.04$ & $1.28 \pm 0.03$ \\
\hline 360 & $0.74 \pm 0.01$ & NA \\
\hline 420 & $0.91 \pm 0.04$ & $1.12 \pm 0.02$ \\
\hline 460 & NA & $1.28 \pm 0.02$ \\
\hline \multicolumn{3}{|c|}{$\begin{array}{l}{ }^{\star 211} \mathrm{At}-\mathrm{IgG} \text { trastuzumab. } \\
+211 \mathrm{At}-\mathrm{MX} 35-\mathrm{F}(\mathrm{ab})_{2} \\
\mathrm{NA}=\text { not applicable. } \\
\text { Data are mean } \pm \mathrm{SD}\end{array}$} \\
\hline
\end{tabular}

levels in the sagittal plane were acquired (Fig. 5). These images revealed elevated activity uptake in several organs known to accumulate free ${ }^{211} \mathrm{At}$, such as the thyroid, salivary gland, and stomach. The lungs and spleen also showed elevated activity uptake in other sections (data not shown). Interestingly, a slightly elevated level of uptake was found in localized areas of the spine. Histologic examination identified these areas as cartilage tissue, appearing as bright discs along the spine (Fig. 5).

\section{DISCUSSION}

In the current study, we describe a novel technique for ex vivo bioimaging of $\alpha$-particle-emitting radionuclides in tissue sections. The $\alpha$-camera is a rapid, inexpensive, fully quantitative imaging technique based on digital autoradiography and the principle of scintillation. The spatial resolution was estimated to be at least $35 \mu \mathrm{m}$ and uniformity better than $2 \%$, and importantly, signal intensity was linear to radionuclide activity in the imaged sections. Thus, the $\alpha$-camera permitted assessment of the activity distribution of an $\alpha$-emitter at the suborgan level and could therefore provide useful data for small-scale dosimetry.

The $\alpha$-camera visualized and quantified important differences and temporal changes in activity distributions of astatinated bioconjugates, both in normal tissues and tumors.

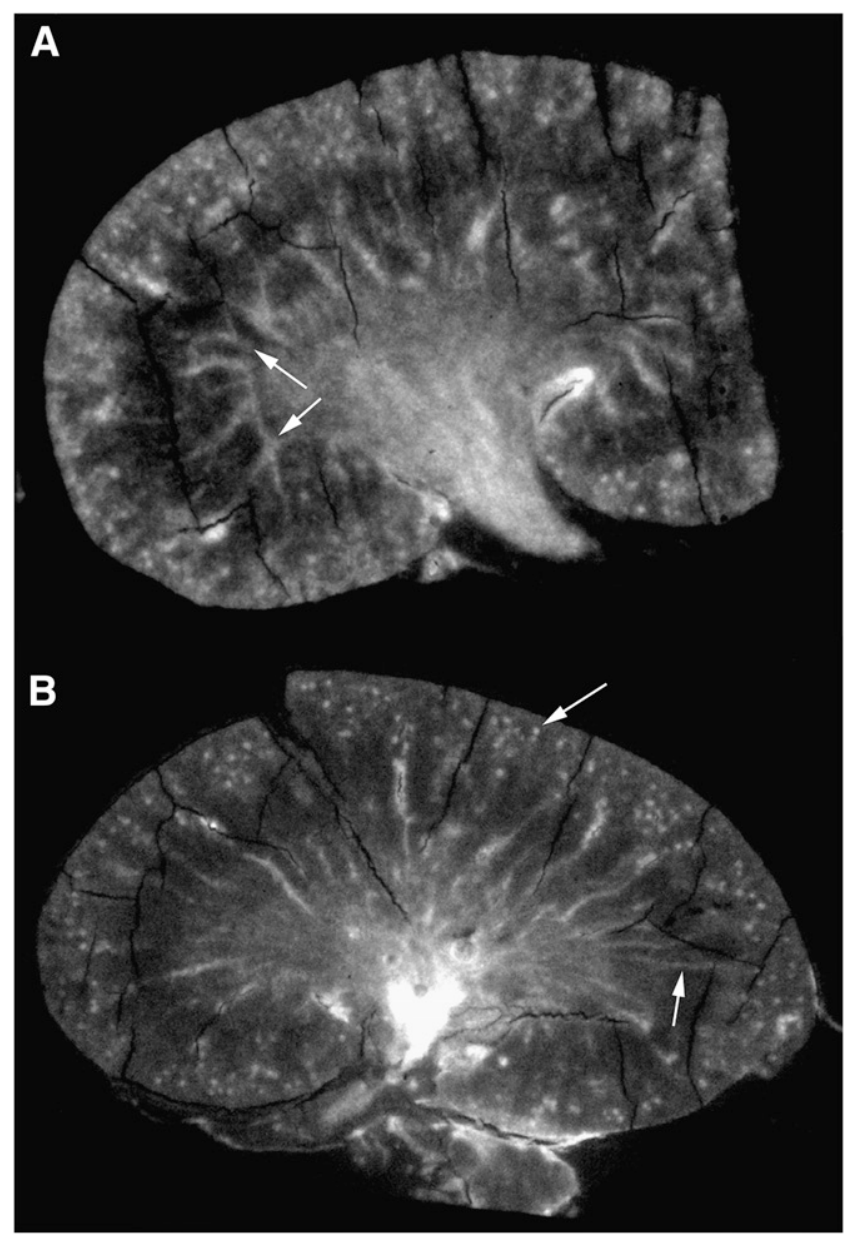

FIGURE 4. Cryosections of mice kidneys imaged with $\alpha$-camera at $30 \mathrm{~min}(\mathrm{~A})$ and $2 \mathrm{~h}(\mathrm{~B})$ after intravenous injection of ${ }^{211} \mathrm{At}-\mathrm{IgG}$ trastuzumab. White arrows indicate vascular branches (A) and medullary rays and glomeruli $(B)$.

Differences in the activity distributions of an $\operatorname{IgG}$ antibody and $\mathrm{a} F\left(\mathrm{ab}^{\prime}\right)_{2}$ fragment were apparent within the renal compartments in studies comparing the 2 radioimmunoconjugates. Although the uptake profile was similar at $20 \mathrm{~min}$ after intravenous injection, a pronounced uptake in the renal cortex was seen for the $F\left(a b^{\prime}\right)_{2}$ fragment after $2 \mathrm{~h}$. This uptake illustrates how estimations and the use of mean absorbed dose from whole-organ biodistribution stud-

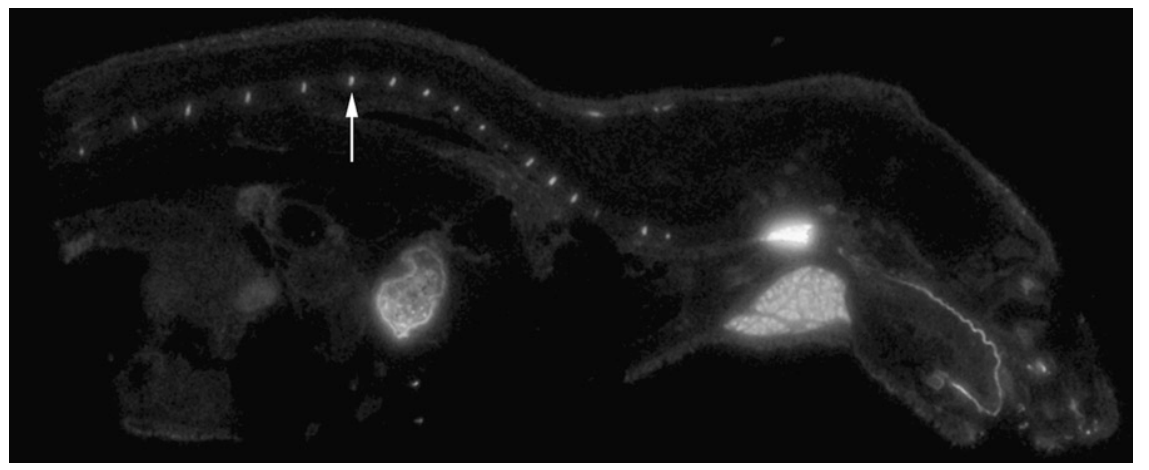

FIGURE 5. Whole-body mouse cryosection imaged with $\alpha$-camera at 30 min after intravenous injection of free ${ }^{211} \mathrm{At}$. White arrow indicates cartilage in spine. 
ies may be misleading for $\alpha$-emitters, because this method fails to estimate the absorbed dose in the renal cortex on a small-scale level. Although it cannot be ruled out that the differences in renal distribution between the IgG antibody (trastuzumab) and the $\mathrm{MX} 35-\mathrm{F}\left(\mathrm{ab}^{\prime}\right)_{2}$ antibody fragment result from characteristics other than molecular size, this comparison exemplifies the utility of the $\alpha$-camera for quantitative studies.

We observed time-dependent changes in activity distribution after the intravenous injection of astatinated MX35$\mathrm{F}\left(\mathrm{ab}^{\prime}\right)_{2}$ fragments by serial imaging of OVCAR-3 tumors from different animals. Images acquired at $10 \mathrm{~min}$ after injection revealed small high-intensity areas throughout the tumor, likely representing high activity levels in vasculature. Markedly nonuniform activity distributions in tumors persisted $7 \mathrm{~h}$ after injection. However, images taken at $21 \mathrm{~h}$ after injection revealed a much more uniform activity distribution, reflecting the deeper bioconjugate penetration that would be more favorable for therapy.

The utility of autoradiography in high-resolution singlecell imaging was appreciated early (25), as was quantitative analysis of autoradiograms (26). Typically, autoradiograms are acquired with the tissue sections in direct contact with the radiation-detecting medium, and therefore the picture scale for each technique is generally fixed. A major advantage of the $\alpha$-camera is the possibility of optically varying the picture scale. The resolution of the imaging system was not limited by the optical registration of the scintillation photons but primarily by the events taking place in the tissue sections and scintillating material. The picture scale could thus be varied by altering the focal distance, allowing imaging of multiple or large specimens with only a minor decrease in pixel resolution.

The $\alpha$-camera is currently limited by the fact that the same tissue section cannot be used for both imaging and morphologic identification, because the dry-mounting on the scintillator excludes further histologic processing of that section. Instead, a consecutive section before or after the imaged section must be used for H\&E staining and overlay with the imaged section. Even though major landmarks such as peripheral borders can be aligned in the overlay procedure, differential adherence of cryosections to microscopy slides as opposed to the scintillator can result in spatial displacement and imperfect superimposition. This problem may be solved using image software that allows spatial displacement of the histologic image in the overlay procedure. Initial attempts to overlay kidney images with H\&E sections identified renal structures such as glomeruli, medullary rays, and vascular branches stretching toward the renal cortex. In tumor sections of OVCAR-3 xenografts, the higher-intensity ring-shaped structures were identified as stroma surrounding clusters of tumor cells. The tumor images shown in this study illustrate the different temporal distribution stages for antibody penetration in a tumor, including extravasation and diffusion with binding, but they are not intended to provide a comprehensive analysis.
Many studies $(10,12,27-29)$ have shown how quantitative autoradiography could improve dosimetry models and estimations within targeted $\alpha$-radiotherapy. Future studies with the $\alpha$-camera will include dosimetry modeling and estimations. The $\alpha$-camera can assist dosimetry by identifying potential source regions, target volumes, and target dimensions. The time-dependent changes in activity distribution can be studied from serial images, providing important data on the input parameters needed for small-scale dosimetry.

\section{CONCLUSION}

This study presents a novel, portable imaging technology dedicated to the ex vivo detection and activity distribution assessment of $\alpha$-particles in tissues at the suborgan level. This digital autoradiographic technique uses optical imaging of cryosections transferred onto a scintillator, providing a tool for rapid and quantitative bioimaging of $\alpha$-emitting radionuclides in tissues. The $\alpha$-camera measures the activity distribution down to a scale approaching the cellular level by virtue of the short path lengths of $\alpha$-particles, which also contribute to the high resolution ( $35 \mu \mathrm{m}$ or less) of the method. The $\alpha$-camera can be used for several important purposes: to identify dose-limiting normal organs with nonuniform activity distributions (requiring small-scale dosimetry to accurately predict biologic outcomes); compare different bioconjugates (e.g., molecular sizes) to improve favorable tumor distributions or avoid unfavorable normal-tissue distributions; and develop small-scale dosimetry models that can estimate the probabilities of tumor control and normal-tissue toxicities, based on more relevant and accurate estimations of absorbed dose. In summary, the characteristics of this bioimaging system are promising, and we propose that the $\alpha$-camera can become an important tool to develop and evaluate future targeted $\alpha$-particle radiotherapy.

\section{ACKNOWLEDGMENTS}

We thank Sture Lindegren for help with radiolabeling, Magnus Båth for consultations in digital imaging, Roland D'Argy and Karin Hallbäck for whole-body sectioning, and Agne Larsson for initiating this project. We also thank Ragnar Hultborn and Oliver Press for valuable help with the manuscript. This work was supported by grants from the Swedish Cancer Society, Swedish Research Council, Swedish Radiation Safety Authority, Assar Gabrielsson Foundation, and the King Gustaf V Jubilee Clinic Research Foundation in Gothenburg, Sweden.

\section{REFERENCES}

1. Andersson H, Elgqvist J, Horvath G, et al. Astatine-211-labeled antibodies for treatment of disseminated ovarian cancer: an overview of results in an ovarian tumor model. Clin Cancer Res. 2003;9:3914S-3921S.

2. Back T, Andersson H, Divgi CR, et al. ${ }^{211}$ At radioimmunotherapy of subcutaneous human ovarian cancer xenografts: evaluation of relative biologic effectiveness of an $\alpha$-emitter in vivo. J Nucl Med. 2005;46:2061-2067. 
3. Elgqvist J, Andersson H, Back T, et al. $\alpha$-radioimmunotherapy of intraperitoneally growing OVCAR-3 tumors of variable dimensions: outcome related to measured tumor size and mean absorbed dose. J Nucl Med. 2006;47:1342-1350.

4. Elgqvist J, Andersson H, Back T, et al. Therapeutic efficacy and tumor dose estimations in radioimmunotherapy of intraperitoneally growing OVCAR-3 cells in nude mice with ${ }^{211}$ At-labeled monoclonal antibody MX35. J Nucl Med. 2005; 46:1907-1915.

5. Zalutsky MR. Targeted $\alpha$-particle therapy of microscopic disease: providing a further rationale for clinical investigation. J Nucl Med. 2006;47:12381240 .

6. Drecoll E, Gaertner FC, Miederer M, et al. Treatment of peritoneal carcinomatosis by targeted delivery of the radio-labeled tumor homing peptide bi-DTPA-[F3]2 into the nucleus of tumor cells. PLOS ONE. 2009;4:e5715.

7. Andersson H, Cederkrantz E, Back T, et al. Intraperitoneal $\alpha$-particle radioimmunotherapy of ovarian cancer patients: pharmacokinetics and dosimetry of ${ }^{211}$ At-MX35 $\mathrm{F}\left(\mathrm{ab}^{\prime}\right) 2$ : a phase I study. J Nucl Med. 2009;50:11531160 .

8. Zalutsky MR, Reardon DA, Akabani G, et al. Clinical experience with $\alpha$-particle emitting ${ }^{211}$ At: treatment of recurrent brain tumor patients with ${ }^{211}$ At-labeled chimeric antitenascin monoclonal antibody 81C6. J Nucl Med. 2008;49: 30-38.

9. Humm JL, Cobb LM. Nonuniformity of tumor dose in radioimmunotherapy. J Nucl Med. 1990;31:75-83.

10. Humm JL, Macklis RM, Bump K, Cobb LM, Chin LM. Internal dosimetry using data derived from autoradiographs. J Nucl Med. 1993;34:1811-1817.

11. Humm JL, Roeske JC, Fisher DR, Chen GT. Microdosimetric concepts in radioimmunotherapy. Med Phys. 1993;20:535-541.

12. Akabani G, Kennel SJ, Zalutsky MR. Microdosimetric analysis of alpha-particleemitting targeted radiotherapeutics using histological images. J Nucl Med. 2003;44:792-805

13. Wood RW. The scintillations of radium. Science. 1904;29:195-196.

14. Kolar ZI, Den Hollander W. 2003: a centennial of spinthariscope and scintillation counting. Appl Radiat Isot. 2004;61:261-266.

15. Lindegren S, Andersson H, Back T, Jacobsson L, Karlsson B, Skarnemark G. Highefficiency astatination of antibodies using $\mathrm{N}$-iodosuccinimide as the oxidising agent in labelling of $\mathrm{N}$-succinimidyl 3-(trimethylstannyl)benzoate. Nucl $\mathrm{Med}$ Biol. 2001;28:33-39.
16. Lindegren S, Back T, Jensen HJ. Dry-distillation of astatine-211 from irradiated bismuth targets: a time-saving procedure with high recovery yields. Appl Radiat Isot. 2001;55:157-160.

17. Welshinger M, Yin BW, Lloyd KO. Initial immunochemical characterization of MX35 ovarian cancer antigen. Gynecol Oncol. 1997;67:188-192.

18. Yin BW, Kiyamova R, Chua R, et al. Monoclonal antibody MX35 detects the membrane transporter NaPi2b (SLC34A2) in human carcinomas. Cancer Immun. 2008;8:3-11.

19. Thurber GM, Schmidt MM, Wittrup KD. Factors determining antibody distribution in tumors. Trends Pharmacol Sci. 2008;29:57-61.

20. Larsen RH, Murud KM, Akabani G, Hoff P, Bruland OS, Zalutsky MR. ${ }^{211}$ At- and ${ }^{131}$ I-labeled bisphosphonates with high in vivo stability and bone accumulation. J Nucl Med. 1999;40:1197-1203.

21. Wilbur DS, Thakar MS, Hamlin DK, et al. Reagents for astatination of biomolecules. 4. Comparison of maleimido-closo-decaborate(2-) and meta-[ $\left.{ }^{211} \mathrm{At}\right] \mathrm{as}-$ tatobenzoate conjugates for labeling anti-CD45 antibodies with $\left[{ }^{211} \mathrm{At}\right]$ astatine. Bioconjug Chem. 2009;20:1983-1991.

22. Vaidyanathan G, Affleck DJ, Bigner DD, Zalutsky MR. $N$-succinimidyl 3-[ $\left.{ }^{211} \mathrm{At}\right]$ astato-4-guanidinomethylbenzoate: an acylation agent for labeling internalizing antibodies with alpha-particle emitting ${ }^{211}$ At. Nucl Med Biol. 2003;30:351-359.

23. d'Argy R, Sundwall A. Quantitative whole-body radioluminography: future strategy for balance and tissue distribution studies. Regul Toxicol Pharmacol. 2000;31:S57-S62.

24. Ullberg S. Studies on the distribution and fate of S35-labelled benzylpenicillin in the body. Acta Radiol Suppl. 1954;118:1-110.

25. Bayley ST. Autoradiography of single cells. Nature. 1947;160:193-194.

26. Branson H, Hansborough LA. The quantitative theory of autoradiography illustrated through experiments with P32 in the chick embryo. Science. 1948;108:327328.

27. Larsen RH, Bruland OS. Intratumour injection of immunoglobulins labelled with the alpha-particle emitter ${ }^{211} \mathrm{At}$ : analyses of tumour retention, microdistribution and growth delay. Br J Cancer. 1998;77:1115-1122.

28. Neti PV, Howell RW. Log normal distribution of cellular uptake of radioactivity: implications for biologic responses to radiopharmaceuticals. J Nucl Med. 2006; 47:1049-1058.

29. Neti PV, Howell RW. Lognormal distribution of cellular uptake of radioactivity: statistical analysis of $\alpha$-particle track autoradiography. J Nucl Med. 2008; 49:1009-1016. 